\title{
Identification of nucleotide sequence involved in Weissellicin L production
}

\author{
Yi-sheng Chen ${ }^{*}$, Yun-shien Lee, Hui-chung Wu, Chih-ming Chiang, Shwu-fen Pan and Kun-hon Leong
}

\begin{abstract}
Background: Weissellicin L, a novel bacteriocin produced by Weissella hellenica 4-7, was previously characterized but its full amino acid sequence remain unknown. The draft genome sequencing analysis of Weissella hellenica 4-7 was performed and the open reading frame (ORF) encoding the weissellicin $L$ was identified and clarified.

Findings: The obtained results indicated that the mature bacteriocin consists of 29 amino acid residues with a molecular weight of approximately 3205.64 Da. A conserved processing site of two glycine residues in positions -1 and -2 was observed in the leader peptides. The possibility that bacteriocin secretion depended on ATP-binding cassette $(A B C)$ transporter was therefore suggested. Furthermore, primers were designed from $5^{\prime}$ and $3^{\prime}$ flanking sequences of the weissellicin $L$ structural gene. PCR presented a single product and was useful to detect weissellicin $L$ structural gene.
\end{abstract}

Conclusions: To our knowledge, this is the first report describing the full amino acid sequence of Weissellicin L. A rapid method to detect weissellicin $L$ structural gene was also reported in this study.

Keywords: Weissellicin L; Weissella hellenica; Bacteriocin

\section{Introduction}

It has been frequently found that parts of lactic acid bacteria (LAB) strains produce proteinaceous antibacterial compounds, termed as bacteriocins. Many bacteriocins show great inhibitory ability against food pathogens and therefore attract special interest (Klaenhammer 1988; Ennahar et al. 1999; Cleveland et al. 2001; Yang et al. 2014). In the previous studies, we reported that Weissella hellenica 4-7, isolated from the traditional Taiwanese fermented food sian-sianzih (fermented clams), is capable of producing a novel bacteriocin, termed weissellicin $\mathrm{L}$ (Leong et al. 2013).

Several characteristics of weissellicin L, such as sensitivities to enzymes and heat, inhibition spectra, and partial amino acid sequences, have been previously reported (Leong et al. 2013). Results obtained from mass spectrometry analysis revealed the bacteriocin mass of weissellicin L was approximately 3205.64 Da. However, only 17 amino acid residues from $N$-terminal have been clarified. The objectives of this study are quite simple, 1) to clarify the full amino acid sequence of weissellicin L, and 2) to rapidly

\footnotetext{
* Correspondence: yisheng@mail.mcu.edu.tw

Department of Biotechnology, Ming Chuan University, No. 5, De-Ming Rd., Gui-Shan Township, Taoyuan County 333, Taiwan
}

detect weissellicin L structural gene by using PCR amplification method.

\section{Materials and methods DNA extraction}

Strain $W$. hellenica 4-7 was grown in a modified Glucose Yeast Peptone (GYP) medium under the same conditions previously described by Leong et al. (2013). Genomic DNA was extracted from cells and purified using the Qiagen Blood \& Cell Culture DNA kit (Qiagen, Hilden, Germany) (Aguado-Urda et al. 2011).

\section{Draft genome sequencing analysis}

Illumina GA IIx genome analyzer (Illumina, San Diego, CA) was applied to reveal the genome sequence of $W$. hellenica 4-7. Using de novo Velvet algorithms, short reads obtained were assembled to generate a single chromosome sequence (Chiu et al. 2013). Sequence comparison was carried out using the previously obtained partial $\mathrm{N}$ terminal sequence of weissellicin L (Leong et al. 2013) against the draft genome of $W$. hellenica 4-7 (unpublished). Search for similarity between sequences was performed using NCBI BLAST (http://blast.ncbi.nlm.nih.gov/). 


\section{Design of primers}

Weissellicin L-specific oligonucleotide primers were designed from the $5^{\prime}$ and 3 ' flanking sequences of the weissellicin L structural gene sequence by using NCBI/ Primer-BLAST tool (www.ncbi.nlm.nih.gov/tools/primerblast/) (Figure 1). The primer sequences were as follow: 47bat-SF (5'-GCATTGAAATAAAGCGCACAACA-3') and 4-7bat-SR (5'- TTTGAGGCGCATGACATCAC-3'). The oligonucleotides were synthesized by Genomics BioSci \& Tech Ltd. (New Taipei City, Taiwan).

\section{Weissella strains and PCR conditions}

Besides $W$. hellenica 4-7, strains $W$. hellenica BCRC $80264^{\mathrm{T}}$ obtained from the Bioresource Collection and Research Center (BCRC, Hsinchu, Taiwan) and W. hellenica 203 previously isolated from fermented zoned cerith (Chen et al. 2013), were used as the negative controls. Neither strain BCRC 80264 ${ }^{\mathrm{T}}$ nor strain 203 has bacteriocinproducing ability. Culture conditions of $W$. hellenica strains BCRC $80264^{\mathrm{T}}$ and 203 were the same with strain 4-7. The thermal cycling parameters were an initial denaturation at $95^{\circ} \mathrm{C}$ for $3 \mathrm{~min}$ and $30 \mathrm{~s}$ for subsequent cycles, primer annealing at $59^{\circ} \mathrm{C}$ for $30 \mathrm{~s}$ and primer extension at $72^{\circ} \mathrm{C}$ for $1 \mathrm{~min}$. There were 30 cycles followed by a final extension at $72^{\circ} \mathrm{C}$ for $10 \mathrm{~min}$. The PCR products were visualized on a $2 \%$ agarose gel in $1 \times$ TAE. A 100-bp DNA ladder marker (Genomics BioSci \& Tech Ltd., New Taipei City, Taiwan) was used as the size standard.

\section{Results and discussion}

In our previous study, a new bacteriocin termed weissellicin L was identified in Weissella hellenica 4-7 but only partial $N$-terminal amino acid sequence was observed and the complete sequence remained unknown (Leong et al. 2013). The whole genome of strain Weissella hellenica 4-7 was analyzed afterward to create a draft genome sequence (unpublished). The previously identified partial $\mathrm{N}$-terminal amino acid sequence of weissellicin $\mathrm{L}, \mathrm{NH}_{2}-$ KGFLSWASKATSWLVGP, was applied to search against the draft genome of $W$. hellenica 4-7. An open reading frame was detected to match the partial sequence of weissellicin L completely.

The deduced bacteriocin comprised 52 amino acid residues in the full length precursor peptide and 29 residues in the mature peptide (Figure 1). The molecular weight of the deduced 29 amino acid residues was calculated to be 3205.76 Da using Compute pl/Mw tool in the ExPASy Proteomics Server (http://web.expasy.org/compute_pi/). This calculated molecular weight corresponded to the previously determined molecular weight of 3205.64 Da using MALDI-TOF MS (Leong et al. 2013). Therefore, the nucleotide sequences encoding the putative structural gene for weissellicin $\mathrm{L}$ and its flanking regions were revealed. The sequences determined in this study have been deposited in the DDBJ database with accession number AB983710.

The activity of a dedicated ATP-binding cassette (ABC) transporter is required for the secretion of many class II bacteriocin in Gram-positive bacteria (Michiels et al. 2001). A double-glycine-type leader could always be observed with two glycine residues located at positions -1 and -2 of the leader peptides. This double-glycine sequence is a hallmark of the class II bacteriocins exported through ABC transporters (Dimov et al. 2005; Michiels et al. 2001). In this study, the double-glycine-type leader sequence

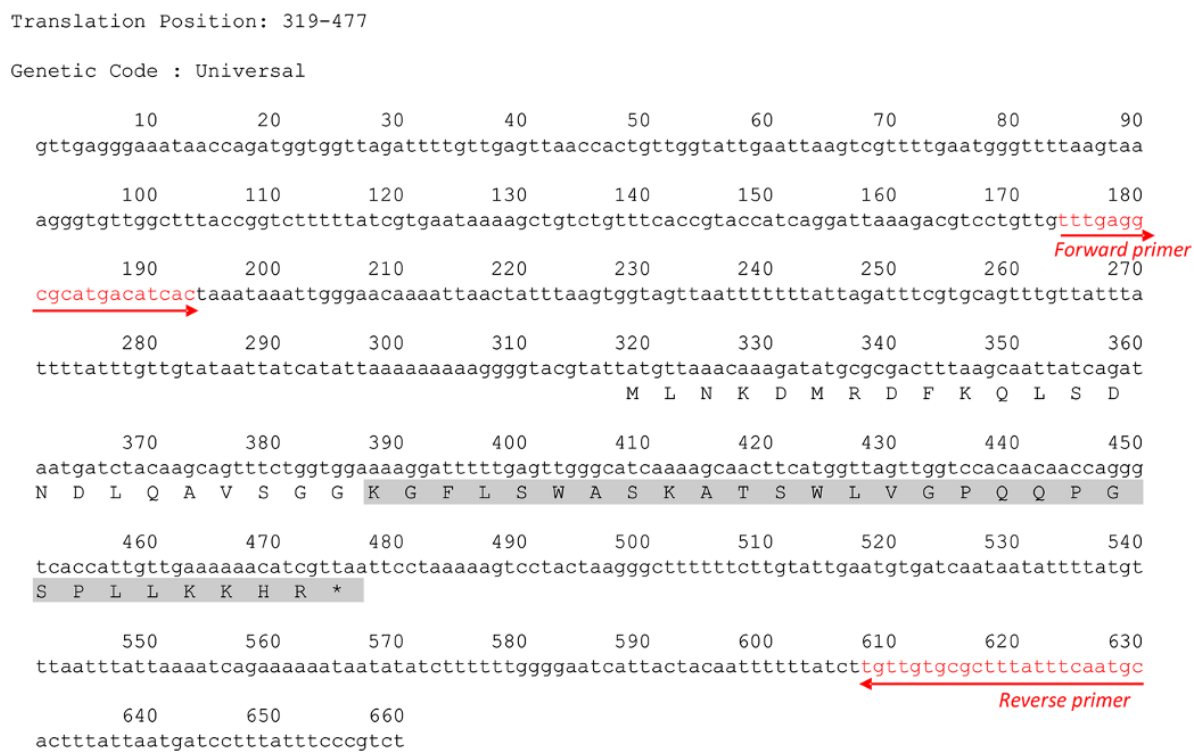

Figure 1 Nucleotide sequence of the weissellicin $L$ gene and the deduced amino acid sequence. An asterisk indicates the translation stop site. The mature weissellicin $L$ peptide is highlighted in grey. 
also presented at the same positions of the leader peptide (Figure 1). It is therefore suggested that weissellicin L was exported through $\mathrm{ABC}$ transporters in W. hellenica 4-7. However, without indepth study the molecular interaction and detailed mechanism of the secretion is not discussed here.

Primers specific for the weissellicin $\mathrm{L}$ gene were designed to perform PCR amplification. A single 457-bp fragment was amplified from the genomic DNA of $W$. hellenica 4-7 (Figure 2). However, no amplified PCR product was observed from W. hellenica strains BCRC $80264^{\mathrm{T}}$ and 203 (Figure 2). PCR product from W. hellenica 4-7 was sequenced and the nucleotide sequences encoding the target gene was confirmed to be identical as reported (Figure 1). The results suggested that PCR amplification with the weissellicin L-specific primers could be useful to select potential weissellicin L-producing strain from multitudinous $W$. hellenica strains.

Our results report the full amino acid sequences of weissellicin $\mathrm{L}$ and the nucleotide sequences encoding the

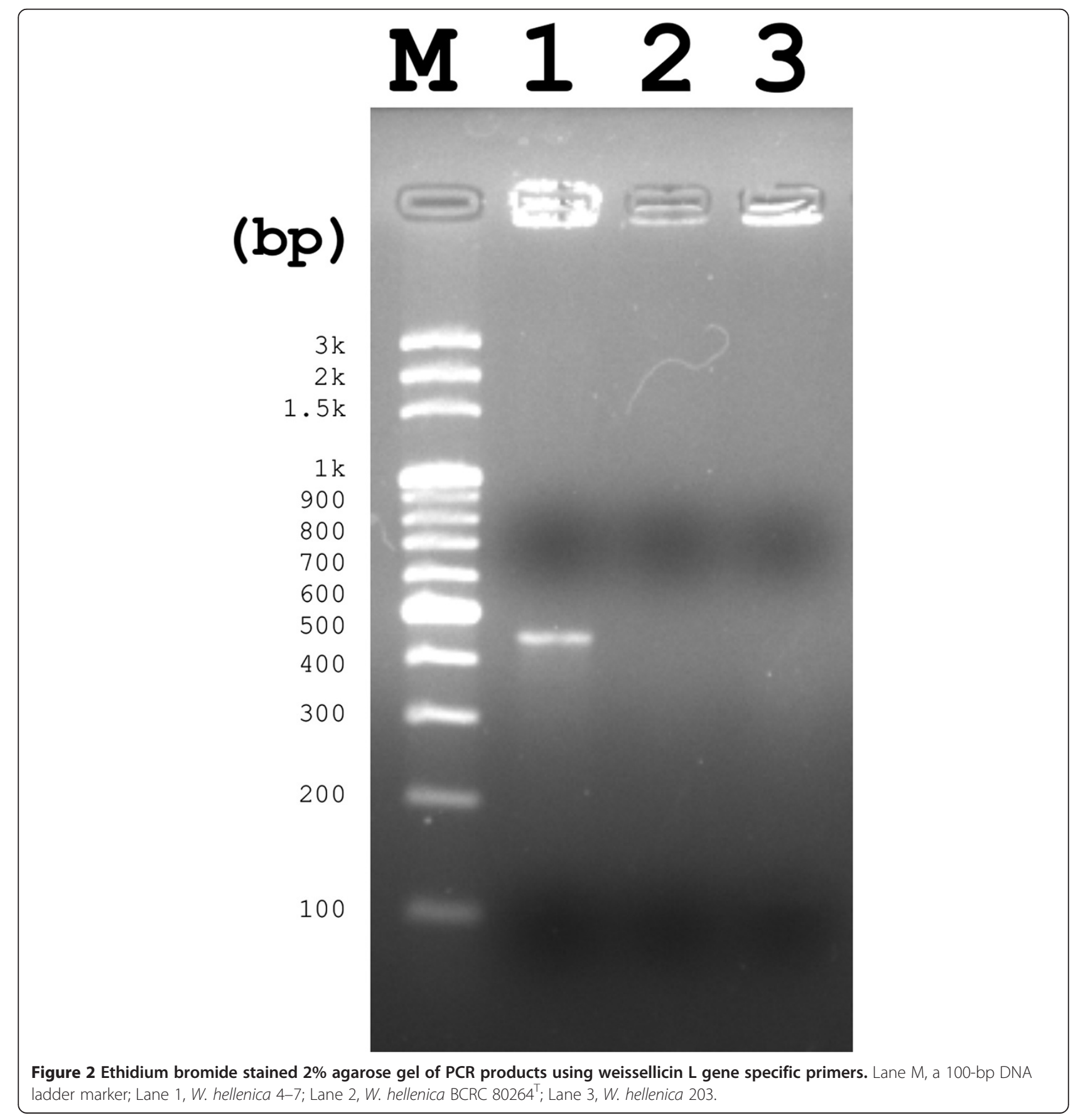


weissellicin L gene. In addition, this study provides a quick method to screening the weissellicin L-producing strain. Further analyses on the genome sequences of $W$. hellenica 4-7 are necessary to understand more bacteriocin related information and other characteristics of LAB.

\section{Competing interest}

The authors declare that they have no competing interests.

\section{Authors' contributions}

YSC, YSL, HCW, SFP and CMC initiated and designed the study. YSC collected the data and drafted manuscript. YSL analyzed the data of draft genome. HCW complete the final manuscript. KHL preformed the DNA experiments. All authors read and approved the final manuscript.

\section{Acknowledgements}

This work was supported by the National Core Facility Program for Biotechnology (Bioinformatics Consortium of Taiwan; NSC 100-2319-B-010-002) for statistic assistance. We also thank the National Science Council Taiwan for financially supporting this study, under Contract No. NSC 102-2313-B-130-00 grant to Yi-sheng Chen.

Received: 9 September 2014 Accepted: 14 October 2014

Published: 18 October 2014

\section{References}

Aguado-Urda M, López-Campos GH, Blanco MM, Fernández-Garayzábal JF, Cutuli MT, Aspiroz C, López-Alonso V, Gibello A (2011) Genome sequence of Lactococcus garvieae 21881, isolated in a case of human septicemia. J Bacteriol 193:4033-4034

Chen YS, Wu HC, Lo HY, Hsu WH, Lin WC, Lin BY (2013) Isolation, identification and characterization of lactic acid bacteria from shao-jiou-luo (fermented zoned cerith), a traditional fermented food in Taiwan. J Aquat Food Prod T 22:543-550

Chiu CM, Chang CH, Pan SF, Wu HC, Li SW, Chang CH, Lee YS, Chiang CM, Chen YS (2013) Draft Genome Sequence of Lactobacillus pobuzihii E100301'. Genome Announc 1(3):e00185-13

Cleveland J, Montville TJ, Nes IF, Chikindas ML (2001) Bacteriocins: safe, natural antimicrobials for food preservation. A review. Int J Food Microbiol 71:1-20

Dimov S, Ivanova P, Harizanova N (2005) Genetics of bacteriocins biosynthesis by lactic acid bacteria. A review. Biotechnol Biotechnol Eq 19:4-10

Ennahar S, Sonomoto K, Ishizaki A (1999) Class lla bacteriocins from lactic acid bacteria: antibacterial activity and food preservation. J Biosci Bioeng 87:705-716

Klaenhammer TR (1988) Bacteriocins of lactic acid bacteria. Biochimie 70:337-349 Leong KH, Chen YS, Lin YH, Pan SF, Yu B, Wu HC, Yanagida F (2013) Weissellicin $\mathrm{L}$, a novel bacteriocin from sian-sianzih-isolated Weissella hellenica 4-7. J Appl Microbiol 115:70-76

Michiels J, Dirix G, Vanderleyden J, Xi C (2001) Processing and export of peptide pheromones and bacteriocins in Gram-negative bacteria. Trends Microbiol 9:164-168

Yang SC, Lin CH, Sung CT, Fang JY (2014) Antibacterial activities of bacteriocins: application in foods and pharmaceuticals. Front Microbiol 5:241

doi:10.1186/2193-1801-3-617

Cite this article as: Chen et al:: Identification of nucleotide sequence involved in Weissellicin L production. SpringerPlus 2014 3:617.

\section{Submit your manuscript to a SpringerOpen ${ }^{\circ}$ journal and benefit from:}

- Convenient online submission

- Rigorous peer review

- Immediate publication on acceptance

- Open access: articles freely available online

- High visibility within the field

- Retaining the copyright to your article

Submit your next manuscript at $>$ springeropen.com 